\title{
ON DIFFERENTIABILITY OF MINIMAL SURFACES AT A BOUNDARY POINT ${ }^{1}$
}

\author{
TUNC GEVECI
}

\begin{abstract}
Let $F(z)=\{u(z), v(z), w(z)\},|z|<1$, represent a minimal surface spanning the curve $\Gamma:\{U(s), V(s), W(s)\}, s$ being the arc length. Suppose $\Gamma$ has a tangent at a point $P$. Then $F(z)$ is differentiable at this point if $U^{\prime}(s), V^{\prime}(s), W^{\prime}(s)$ satisfy a Dini condition at $P$.
\end{abstract}

Let $\Gamma$ be a closed rectifiable Jordan curve in Euclidean 3-space, and let $F(z)=\{u(z), v(z), w(z)\}$, defined in the disk $\{z:|z| \leqq 1\} \quad(z=x+i y$ $\left.=r e^{i \theta}\right)$, represent a generalized minimal surface spanning $\Gamma$, i.e.

(i) $u(z), v(z), w(z)$ are harmonic in $|z|<1$ and continuous in $|z| \leqq 1$;

(ii) $x, y$ are isothermal parameters in $|z| \leqq 1$, i.e.

$$
\begin{aligned}
& \left|F_{x}\right|^{2}:=u_{x}^{2}+v_{x}^{2}+w_{x}^{2}=\left|F_{y}\right|^{2}:=u_{y}^{2}+v_{y}^{2}+w_{y}^{2}, \\
& F_{x} \cdot F_{y}:=u_{x} u_{y}+v_{x} v_{y}+w_{x} w_{y}=0
\end{aligned}
$$

(iii) $F\left(e^{i \theta}\right), 0 \leqq \theta<2 \pi$, is a homeomorphism of $|z|=1$ with $\Gamma$.

The components $u, v, w$ of the vector $F$ are the real parts of analytic functions in $|z|<1$ :

$$
\lambda(z)=u(z)+i u^{*}(z), \quad \mu(z)=v(z)+i v^{*}(z), \quad \nu(z)=w(z)+i w^{*}(z) .
$$

Recently various theorems dealing with the boundary behavior of conformal maps in the plane have been extended to minimal surfaces by J. C. C. Nitsche [2], D. Kinderlehrer [1], S. E. Warschawski [3], and other authors. Nitsche's paper contains a survey of prior work on the boundary behavior of minimal surfaces. The purpose of this note is to present a local result concerning differentiability of minimal surfaces at a given point on the boundary. In fact, our result extends a theorem of Warschawski on conformal mapping in the plane, namely Theorem 1 in [4].

Theorem. Suppose $\{U(s), V(s), W(s)\}$ denotes the parametric representation of $\Gamma$ in terms of arc length. Assume $P_{0}=\left\{U\left(s_{0}\right), V\left(s_{0}\right), W\left(s_{0}\right)\right\}$ is a point of $\Gamma$ and that $\Gamma$ has a tangent at $P_{0}$, i.e. $U^{\prime}\left(s_{0}\right), V^{\prime}\left(s_{0}\right), W^{\prime}\left(s_{0}\right)$ exist. ${ }^{2}$

Received by the editors May 8, 1970.

AMS 1969 subject classifications. Primary 5304, 3040, 3062.

Key words and phrases. Complex analysis, minimal surfaces, boundary behavior.

1 Research supported in part by U. S. Air Force Grant AFOSR-68-1514.

${ }^{2}$ We assume $\left\{U^{\prime}\left(s_{0}\right), V^{\prime}\left(s_{0}\right), W^{\prime}\left(s_{0}\right)\right\}$ represents the unit tangent to $\Gamma$ at $P_{0}$. 
Suppose that there exists a nondecreasing, continuous function $\omega(t) \geqq 0,0 \leqq t \leqq a(a>0)$, such that

$$
\int_{0}^{a} \frac{\omega(t)}{t} d t<\infty
$$

and

$$
\begin{aligned}
\left|U^{\prime}(s)-U^{\prime}\left(s_{0}\right)\right| & \leqq \omega\left(\left|s-s_{0}\right|\right), \\
\left|V^{\prime}(s)-V^{\prime}\left(s_{0}\right)\right| & \leqq \omega\left(\left|s-s_{0}\right|\right), \\
\left|W^{\prime}(s)-W^{\prime}\left(s_{0}\right)\right| & \leqq \omega\left(\left|s-s_{0}\right|\right),
\end{aligned}
$$

for all points $\{U(s), V(s), W(s)\}$ in a neighborhood of $P_{0}$ at which $U^{\prime}(s), V^{\prime}(s), W^{\prime}(s)$ exist. ${ }^{3}$

Let $F\left(e^{i \theta_{0}}\right)=P_{0}$. Then

$$
\lim _{z \rightarrow z_{0}} \frac{\lambda(z)-\lambda\left(z_{0}\right)}{z-z_{0}}=\lambda^{\prime}\left(z_{0}\right) \quad\left(z_{0}=e^{i \theta_{0}}\right)
$$

exists for unrestricted approach in $|z| \leqq 1\left(z \neq z_{0}\right)$, and

$$
\lim _{z \rightarrow z_{0}} \lambda^{\prime}(z)=\lambda^{\prime}\left(z_{0}\right)
$$

for $z$ in any Stolz angle with vertex at $z_{0}$. The same holds for $\mu(z)$ and $\nu(z) .^{4}$

Proof. Without loss of generality we may assume $U^{\prime}\left(s_{0}\right)=1$, $V^{\prime}\left(s_{0}\right)=0, W^{\prime}\left(s_{0}\right)=0$. Under the conditions of the theorem Warschawski proved the following facts (see [3, Part II, §§2-7]):

There is an interval $\left[\theta_{1}, \theta_{2}\right]$ containing $\theta_{0}$ in its interior, a constant $\alpha>1$, and a sector $S=\left\{z=r e^{i \theta}: 0<r<1, \theta_{1}<\theta<\theta_{2}\right\}$ such that, if $\varphi(\zeta)$ maps $|\zeta|<1$ conformally onto $S\left(\varphi(1)=e^{i \theta_{0}}\right)$ and

$$
\tilde{f}=\log \left[\left(\lambda_{\theta}+\alpha\right) \circ \varphi\right]=\log \left[\tilde{\lambda}_{\theta}+\alpha\right] \quad\left(\lambda_{\theta}=\frac{\partial \lambda\left(r e^{i \theta}\right)}{\partial \theta}\right),
$$

then $\lim _{\zeta \rightarrow 1} \operatorname{Im} \tilde{f}(\zeta)$ exists for unrestricted approach in $|\zeta| \leqq 1$ as well as $\lim _{\rho \uparrow 1} \tilde{f}(\rho)=\tilde{f}(1)$. The same holds for $i \tilde{g}=i\left[\mu_{\theta} /\left(\lambda_{\theta}+\alpha\right)\right] \circ \varphi$ $=i \cdot\left[\tilde{\mu}_{\theta} /\left(\bar{\lambda}_{\theta}+\alpha\right)\right]$ and $i \hbar=i \cdot\left[\nu_{\theta} /\left(\lambda_{\theta}+\alpha\right)\right] \circ \varphi=i \cdot\left[\tilde{\nu}_{\theta} /\left(\hat{\lambda}_{\theta}+\alpha\right)\right] .{ }^{5} \operatorname{Let} \Phi(\zeta)$

${ }^{3}$ It should be noted that under the hypotheses of the theorem one can show the existence of a subarc $\gamma$ containing $P_{0}$ in its interior and having the following property: $\Delta s \leqq c\left(P_{1} P_{2}\right)^{-}$where $c$ is a constant, $c>1, \Delta s$ is the length of the subarc of $\gamma$ between $P_{1}, P_{2} \in \gamma$, and $\left(P_{1} P_{2}\right)$ is the chordal distance.

4 The author wishes to express his indebtedness to the referee for his remark simplifying the statement of the theorem.

${ }^{5}$ Isothermal relations (1) and (2) are essential in obtaining these results. 
$=\Phi_{1}(\zeta)+i \Phi_{2}(\zeta)\left(\Phi_{1}=\operatorname{Re} \Phi, \Phi_{2}=\operatorname{Im} \Phi\right)$ be holomorphic in $|\zeta|<1$. Assume

$$
\lim _{\rho \uparrow 1} \Phi(\rho)=\Phi(1)=\Phi_{1}(1)+i \Phi_{2}(1)
$$

exists, and

$$
\lim _{\zeta \rightarrow 1} \Phi_{2}(\zeta)=\Phi_{2}(1)
$$

for unrestricted approach in $|\zeta|<1$. Then by a theorem of Warschawski $[5$, p. 315 , Theorem II $]$ one has

$$
\lim _{\eta \rightarrow 0} \frac{1}{\eta} \int_{0}^{\eta}\left\{\exp \left[\Phi\left(e^{i t}\right)-\Phi(1)\right]\right\} e^{i t} d t=1
$$

and

$$
\lim _{\eta \rightarrow 0} \frac{1}{\eta} \int_{0}^{\eta}\left\{\exp \left[\Phi_{1}\left(e^{i t}\right)-\Phi_{1}(1)\right]-1\right\} d t=0 .
$$

Also, it is readily seen from the proof of this theorem that

There exists a subarc $\tilde{\gamma}$ of $|\zeta|=1$ with midpoint $\zeta=1$

(iii) such that $\lim _{\rho \uparrow 1} \Phi\left(\rho e^{i t}\right)=\Phi\left(e^{i t}\right)$ exists for almost all $e^{i t} \in \tilde{\gamma}$, $\Phi\left(e^{i t}\right)$ is integrable along $\tilde{\gamma}$, and

(7) (iv) $\lim _{\eta \rightarrow 0} \frac{1}{\eta} \int_{0}^{\eta}\left|\Phi\left(e^{i t}\right)-\Phi(1)\right|^{2} d t=0$.

Since $\varphi^{\prime}(\zeta) \neq 0$, we can define $\log \varphi^{\prime}(\zeta)$ as a single valued analytic function in $|\zeta|<1$. By our remarks at the beginning, $\Phi_{0}(\zeta)=\tilde{f}(\zeta)+$ $\log \varphi^{\prime}(\zeta)$ satisfies (3) and (4) and we can apply (5) to $\Phi_{0}(\zeta)$ to obtain

$$
\begin{aligned}
\lim _{\eta \rightarrow 0} \frac{1}{\eta} \int_{0}^{\eta}\left\{\operatorname { e x p } \left[\log \left(\tilde{\lambda}_{\theta}\left(e^{i t}\right)+\alpha\right)\right.\right. & +\log \varphi^{\prime}\left(e^{i t}\right) \\
& \left.\left.-\log \left(\tilde{\lambda}_{\theta}(1)+\alpha\right)-\log \varphi^{\prime}(1)\right]\right\} e^{i t} d t=1
\end{aligned}
$$

which implies

$$
\lim _{\eta \rightarrow 0} \frac{1}{\eta} \int_{0}^{\eta} \frac{\left(\tilde{\lambda}_{\theta}\left(e^{i t}\right)+\alpha\right) \varphi^{\prime}\left(e^{i t}\right)}{\left(\tilde{\lambda}_{\theta}(1)+\alpha\right) \varphi^{\prime}(1)} e^{i t} d t=1 .
$$

Letting $\varphi\left(e^{i \eta}\right)=e^{i \xi}$ and changing the variable of integration $\left(\varphi\left(e^{i t}\right)\right.$ $\left.=e^{i \theta}\right)$ we readily obtain 


$$
\lim _{\xi \rightarrow \theta_{0}} \frac{1}{\xi-\theta_{0}} \int_{\theta_{0}}^{\xi}\left(\lambda_{\theta}\left(e^{i \theta}\right)+\alpha\right) e^{i \theta} d \theta=\left(\lambda_{\theta}\left(e^{i \theta 0}\right)+\alpha\right) e^{i \theta 0} .
$$

Now,

$$
\begin{aligned}
& e^{i \theta_{0}}\left[\frac{\lambda\left(e^{i \xi}\right)-\lambda\left(e^{i \theta 0}\right)}{\xi-\theta_{0}}\right] \\
& =\frac{1}{\xi-\theta_{0}} \int_{\theta_{0}}^{\xi} \lambda_{\theta}\left(e^{i \theta}\right)\left(e^{i \theta_{0}}-e^{i \theta}\right) d \theta+\frac{1}{\xi-\theta_{0}} \int_{\theta_{0}}^{\xi} \lambda_{\theta}\left(e^{i \theta}\right) e^{i \theta} d \theta,
\end{aligned}
$$

since $\lambda\left(e^{i \theta}\right)$ is absolutely continuous [ 3 ]. By (8), the second term in (9) approaches the limit $\lambda_{\theta}\left(e^{i \theta_{0}}\right) e^{i \theta_{0}}$, and the first term approaches 0 as $\xi \rightarrow \theta_{0}$, since

$$
\frac{1}{\left|\xi-\theta_{0}\right|} \int_{\theta_{0}}^{\xi}\left|\lambda_{\theta}\left(e^{i \theta}\right)\right|\left|e^{i \theta_{0}}-e^{i \theta}\right| d \theta \leqq \frac{\left|e^{i \xi}-e^{i \theta_{0}}\right|}{\xi-\theta_{0}} \int_{\theta_{0}}^{\xi}\left|\lambda_{\theta}\left(e^{i \theta}\right)\right| d \theta
$$

and $\lambda_{\theta}\left(e^{i \theta}\right)$ is integrable. Therefore,

$$
\lim _{\xi \rightarrow \theta_{0}} \frac{\lambda\left(e^{i \xi}\right)-\lambda\left(e^{i \theta_{0}}\right)}{\xi-\theta_{0}}=\lambda_{\theta}\left(e^{i \theta_{0}}\right) .
$$

From (10) it follows that

$$
\lim _{e^{i \theta \rightarrow e^{i \theta_{0}}}} \frac{\lambda\left(e^{i \theta}\right)-\lambda\left(e^{i \theta_{0}}\right)}{e^{i \theta}-e^{i \theta_{0}}}=\lambda^{\prime}\left(e^{i \theta_{0}}\right)
$$

exists.

The function $\left(\lambda(z)-\lambda\left(e^{i \theta_{0}}\right)\right) /\left(z-e^{i \theta_{0}}\right)$ is holomorphic in $|z|<1$ and by (11) and by the fact that $\lambda(z)$ is continuous on $|z|=1$ it is bounded on $|z|=1$. The continuity of $\lambda(z)$ in $|z| \leqq 1$ also ensures that

$$
\frac{\lambda(z)-\lambda\left(e^{i \theta_{0}}\right)}{z-e^{i \theta_{0}}}=O\left(\frac{1}{\left|z-e^{i \theta_{0}}\right|}\right) \quad \text { for }|z|<1 .
$$

Therefore, by a theorem of Phragmén-Lindelöf

$$
\left(\left(\lambda(z)-\lambda\left(e^{i \theta_{0}}\right)\right) /\left(z-e^{i \theta_{0}}\right)\right.
$$

is bounded in $|z|<1$. Hence, by a theorem of Lindelöf,

$$
\lim _{z \rightarrow e^{i \theta_{0}}} \frac{\lambda(z)-\lambda\left(e^{i \theta_{0}}\right)}{z-e^{i \theta_{0}}}=\lambda^{\prime}\left(e^{i \theta_{0}}\right)
$$

for unrestricted approach in $|z| \leqq 1$. The second equation, 
$\lim _{z \rightarrow z_{0}} \lambda^{\prime}(z)=\lambda^{\prime}\left(z_{0}\right)$ in any Stolz angle with vertex at $z_{0}$, is a wellknown consequence of the first.

We can apply (7) to $i \tilde{g}(\zeta)$ and obtain

$$
\lim _{\eta \rightarrow 0} \frac{1}{\eta} \int_{0}^{\eta}\left|\frac{\tilde{\mu}_{\theta}\left(e^{i t}\right)}{\bar{\lambda}_{\theta}\left(e^{i t}\right)+\alpha}-\frac{\tilde{\mu}_{\theta}(1)}{\tilde{\lambda}_{\theta}(1)+\alpha}\right|^{2} d t=0 .
$$

Also, we can apply (6) to $2 \tilde{f}(\zeta)$ and conclude that

$$
\lim _{\eta \rightarrow 0} \frac{1}{\eta} \int_{0}^{\eta}\left\{\left|\frac{\tilde{\lambda}_{\theta}\left(e^{i t}\right)+\alpha}{\tilde{\lambda}_{\theta}(1)+\alpha}\right|^{2}-1\right\} d t=0 .
$$

Thus,

$$
\frac{1}{|\eta|} \int_{0}^{\eta}\left|\tilde{\lambda}_{\theta}\left(e^{i t}\right)+\alpha\right|^{2} d t \leqq M_{0}
$$

for $|\eta| \leqq \eta_{0}$ and some constant $M_{0}$.

By Schwarz's inequality,

$$
\begin{aligned}
\frac{1}{\eta} \int_{0}^{\eta} \mid \tilde{\mu}_{\theta}\left(e^{i t}\right)\left(\tilde{\lambda}_{\theta}(1)\right. & +\alpha)-\tilde{\mu}_{\theta}(1)\left(\tilde{\lambda}_{\theta}\left(e^{i t}\right)+\alpha\right) \mid d t \\
\leqq & \left(\frac{1}{\eta} \int_{0}^{\eta}\left|\frac{\tilde{\mu}_{\theta}\left(e^{i t}\right)}{\tilde{\lambda}_{\theta}\left(e^{i t}\right)+\alpha}-\frac{\tilde{\mu}_{\theta}(1)}{\tilde{\lambda}_{\theta}(1)+\alpha}\right|^{2} d t\right)^{1 / 2} \\
& \cdot\left(\left|\tilde{\lambda}_{\theta}(1)+\alpha\right|^{2} \cdot \frac{1}{\eta} \int_{0}^{\eta}\left|\tilde{\lambda}_{\theta}\left(e^{i t}\right)+\alpha\right|^{2} d t\right)^{1 / 2} ;
\end{aligned}
$$

(12), (14) and (15) imply

(16) $\lim _{\eta \rightarrow 0} \frac{1}{\eta} \int_{0}^{\eta}\left|\tilde{\mu}_{\theta}\left(e^{i t}\right)\left(\tilde{\lambda}_{\theta}(1)+\alpha\right)-\tilde{\mu}_{\theta}(1)\left(\tilde{\lambda}_{\theta}\left(e^{i t}\right)+\alpha\right)\right| d t=0$.

Since $\varphi^{\prime}\left(e^{i t}\right)$ is bounded in a neighborhood of $\zeta=1$, we also have

$$
\lim _{\eta \rightarrow 0} \frac{1}{\eta} \int_{0}^{\eta}\left|\tilde{\mu}_{\theta}\left(e^{i t}\right)\left(\tilde{\lambda}_{\theta}(1)+\alpha\right)-\tilde{\mu}_{\theta}(1)\left(\tilde{\lambda}_{\theta}\left(e^{i t}\right)+\alpha\right)\right|\left|\phi^{\prime}\left(e^{i t}\right)\right| d t=0 .
$$

Changing the variable of integration, as in the case of $\lambda_{\theta}\left(e^{i \theta}\right)$, one concludes from (17) that

(18) $\lim _{\xi \rightarrow \theta_{0}} \frac{1}{\xi-\theta_{0}} \int_{\theta_{0}}^{\xi}\left|\mu_{\theta}\left(e^{i \theta}\right)\left(\lambda_{\theta}\left(e^{i \theta 0}\right)+\alpha\right)-\mu_{\theta}\left(e^{i \theta 0}\right)\left(\lambda_{\theta}\left(e^{i \theta}\right)+\alpha\right)\right| d \theta=0$.

Thus, 


$$
\begin{aligned}
& \left(\lambda_{\theta}\left(e^{i \theta_{0}}\right)+\alpha\right) \lim _{\xi \rightarrow \theta_{0}} \frac{1}{\xi-\theta_{0}} \int_{\theta_{0}}^{\xi} \mu_{\theta}\left(e^{i \theta}\right) d \theta \\
& \quad=\mu_{\theta}\left(e^{i \theta 0}\right) \lim _{\xi \rightarrow \theta_{0}} \frac{1}{\xi-\theta_{0}} \int_{\theta_{0}}^{\xi}\left(\lambda_{\theta}\left(e^{i \theta}\right)+\alpha\right) d \theta .
\end{aligned}
$$

By (10)

$$
\lim _{\xi \rightarrow \theta_{0}} \frac{1}{\xi-\theta_{0}} \int_{\theta_{0}}^{\xi}\left(\lambda_{\theta}\left(e^{i \theta}\right)+\alpha\right) d \theta=\lambda_{\theta}\left(e^{i \theta_{0}}\right)+\alpha,
$$

and $\lambda_{\theta}\left(e^{i \theta_{0}}\right)+\alpha \neq 0$.

Therefore (19) and (20) imply

$$
\lim _{\xi \rightarrow \theta_{0}} \frac{1}{\xi-\theta_{0}} \int_{\theta_{0}}^{\xi} \mu_{\theta}\left(e^{i \theta}\right) d \theta=\mu_{\theta}\left(e^{i \theta}\right) .
$$

From (21) one infers that

$$
\lim _{z \rightarrow e^{i \theta_{0}}} \frac{\mu(z)-\mu\left(e^{i \theta_{0}}\right)}{z-e^{i \theta_{0}}}
$$

exists for unrestricted approach in $|z| \leqq 1$, exactly the same way as we showed this limit exists in the case of $\lambda(z)$. We deal with $\nu(z)$ in a similar fashion.

It should be noted that one may assume only the subarc $\gamma$ to be rectifiable and obtain the same result with slight modification of our proof.

\section{BIBLIOGRAPHY}

1. D. Kinderlehrer, The boundary regularity of minimal surfaces, Ann. Scuola Norm. Sup. Pisa 23 (1969), 711-744.

2. J. C. C. Nitsche, The boundary behavior of minimal surfaces. Kellogg's theorem and branch points on the boundary, Invent. Math. 8 (1969), 313-333.

3. S. E. Warschawski, Boundary derivatives of minimal surfaces, Arch. Rational Mech. Anal. 38 (1970), 241-256.

4. - On differentiability at the boundary in conformal mapping, Proc. Amer. Math. Soc. 12 (1961), 614-620. MR 24 \#A1374.

5. - On the higher derivatives at the boundary in conformal mapping, Trans. Amer. Math. Soc. 38 (1935), 310-340.

University of California at San Diego, la Jolla, California 92037 